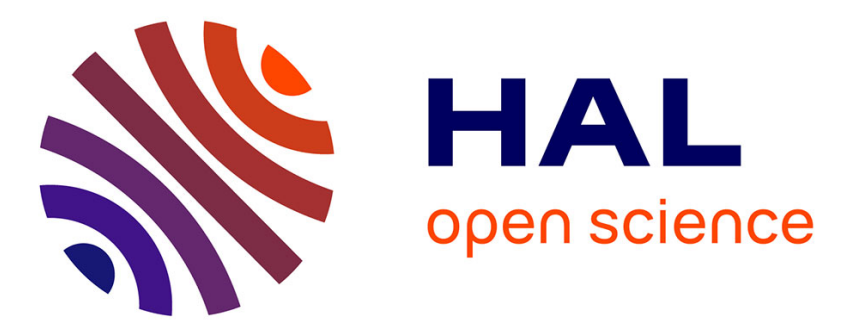

\title{
Characterization of 3D morphology and microcracks in composites reinforced by multi-axial multi-ply stitched preforms
}

Pierre-Jaques Liotier, Alain Vautrin, Christine Delisée

\section{- To cite this version:}

Pierre-Jaques Liotier, Alain Vautrin, Christine Delisée. Characterization of 3D morphology and microcracks in composites reinforced by multi-axial multi-ply stitched preforms. Composites Part A: Applied Science and Manufacturing, 2010, 41 (5), pp.653-662. 10.1016/j.compositesa.2010.01.015 . hal-00850041

\section{HAL Id: hal-00850041 https://hal.science/hal-00850041}

Submitted on 8 Sep 2021

HAL is a multi-disciplinary open access archive for the deposit and dissemination of scientific research documents, whether they are published or not. The documents may come from teaching and research institutions in France or abroad, or from public or private research centers.
L'archive ouverte pluridisciplinaire HAL, est destinée au dépôt et à la diffusion de documents scientifiques de niveau recherche, publiés ou non, émanant des établissements d'enseignement et de recherche français ou étrangers, des laboratoires publics ou privés. 


\title{
Characterization of 3D morphology and microcracks in composites reinforced by multi-axial multi-ply stitched preforms
}

\author{
Pierre-Jacques Liotier ${ }^{\mathrm{a}, *}$, Vautrin Alain ${ }^{\mathrm{a}}$, Delisée Christine ${ }^{\mathrm{b}}$ \\ a École Nationale Supérieure des Mines de Saint-Étienne 158, cours Fauriel, F-42023 SAINT-ÉTIENNE Cedex 2, France \\ ${ }^{\mathrm{b}}$ Unité Sciences du Bois et des Biopolymères, Université Bordeaux 1, Domaine de l'Hermitage, 69, route d'Arcachon, F-33612 CESTAS Cedex, France
}

The micro-structure of polymer matrix composites reinforced by multi-axial multi-ply stitched carbon preforms and manufactured by liquid resin infusion is analyzed. The stitching induces deviations in fibre placement and creates openings which become resin-rich regions after the resin infusion. Characteriza-tion of the size and shape of the resin-rich regions of composites with different stitching yarn size and tightness and various stacking sequences has been performed by $2 \mathrm{D}$ metallographic micrography and X-ray microtomography. The resin-rich region volume was estimated at roughly $3.0 \pm 0.5 \%$ of the material volume. The resin-rich regions constitute about $9 \%$ of the resin in the entire composite, whose fibre vol-ume fraction is close to $65 \%$. X-ray microtomography was successfully used to characterize the 3D micro-cracks created by hygrothermal fatigue.

\section{Introduction}

The combination of the multi-axial multi-ply stitched preforms and the liquid resin infusion (LRI) process appears to offer an efficient way to save weight and cut costs when facing the new challenge of manufacturing thicker and more complex-shaped parts for aircraft.

As a result, composite part manufacturers are actively seeking ways to develop and control Liquid Composite Moulding (LCM) processes, particularly LRI or light Resin Transfer Moulding (RTM). Advanced dry preforms thus need to be designed to meet the new industrial requirements. The Non-Crimp Fabrics (NCF) half-products, multi-axial stacks whose plies are assembled by stitching, are regarded as promising reinforcements in producing complex preforms. The major contribution of stitching is to ease handling and manufacturing efficiency but it does little to enhance the transverse mechanical properties of the final laminates. In contrast with fabrics, it is commonly reported that as the fibres remain relatively straight, NCF fully exploits their superior stiffness and strength. However, it is now admitted that the stitches introduce a complex material morphology inside composite parts. The stitching of the plies produces two main effects (Fig. 1):

- First, fibre in-plane disorientations close to the stitches (herein called "openings").

\footnotetext{
* Corresponding author. Tel.: +33491266215.

E-mail address: pierre-jacques.liotier@univmed.fr (P.-J. Liotier).
}

- Second, the occurrence of two types of resin-rich region at the stitches (herein called "transverse channels") and between the multi-axial stacks due to the stitching yarn loop (herein called “interlayer gaps”).

Several material and processing parameters control these effects, such as stitching yarn size, tension, geometry and physical and mechanical characteristics.

In a multi-axial stack, plies with different fibre orientations are stitched together and the resin-rich regions have complex shapes. These meso-scale resin-rich regions should be characterized before any modelling of the laminate properties. To investigate the morphology of these materials, novel 3D techniques need to be used. Here, classical metallographic methods were used first, giving a rough idea of the material morphology, then X-ray microtomography was applied to provide 3D mapping of the stitching areas inside the laminate volume. However, NCF morphology needs to be characterized, in order to model its mechanical behaviour and any damage that occurs under hygrothermal cyclical fatigue. In other words, 3D microcracking network requires detection. Thus, we report here the first of two experimental approaches aimed at characterizing the 3D hygrothermal microcrack network and its interaction with the resin-rich regions under hygrothermal fatigue. First, we introduce some basic 3D multi-scale characteristics of materials. Then methods are described, with particular attention to the detection of resin-rich regions. Results and discussion follow, with details of the hygrothermal microcracks detected, proving the efficiency of the experimental procedure. 


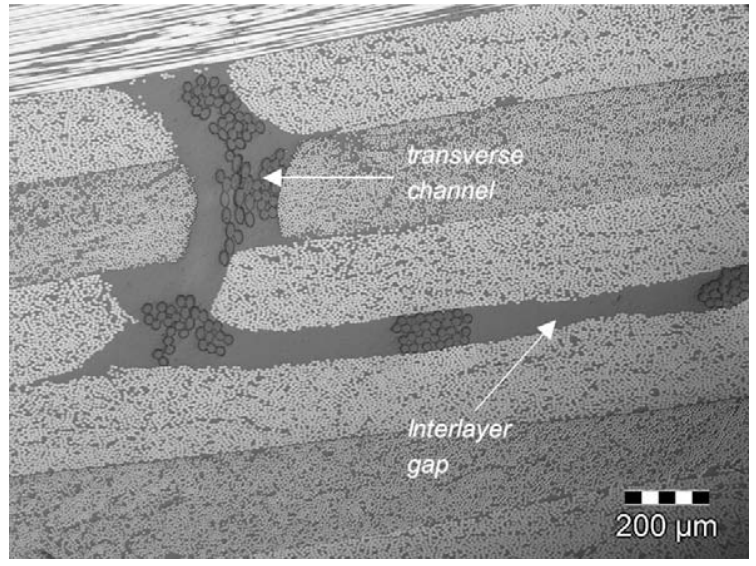

Fig. 1. Transverse channels and interlayer gaps (through-the-thickness section).

\section{Materials}

\subsection{Description}

NCF (Fig. 2a) materials were developed in the 1990's for aeronautic applications. They are manufactured by the continuous deposit of carbon fibre bundles whose sizes are usually within the range $4-24 \mathrm{~K}$ (i.e. 4000 and 24,000 carbon fibres per yarn). The carbon yarns are laid along selected orientations to form stacks which are stitched together (Fig. 3). The stitching yarns are generally polyester yarns that bind the plies together to ease the handling and processing. It is worth noting that the polyester binding yarn usually does not play any special structural role, even though some improvement of the transverse properties of the laminate might be expected. The stitching holds the carbon yarns firmly together; it ensures the regularity of the structure of the preform and controls the mechanical properties of the laminate. While the stitches decrease preform compactness compared to preforms without any resin-rich area (Fig. 1); the stitch density controls the permeability of the dry perform and facilitates resin flow through fibrous reinforcements during the injection or infusion process $[2,3]$.

The stitches introduce resin-rich regions after processing, because of the openings between the carbon fibres close to the stitches (Figs. 1 and 2). The diamond-shaped resin-rich regions create heterogeneous areas inside the plies and may favour new cracking phenomena $[3,4]$. In addition to the transverse channels, other resin-rich regions are located in the interlayer gaps, near the stitching loops.

To improve these multi-axial materials, Hexcel Corporation has developed a novel product, the $\mathrm{NC}^{\circledR}{ }^{\circledR}$ (Non-Crimp New Concept) (Fig. 2b). The main difference is that the fibre yarns are assembled in plies and maintained by a thin hot melt yarn. These layers are cut, oriented in the desired direction and stitched together [3].

The $\mathrm{NC} 2{ }^{\circledR}$ system minimizes the size of the resin-rich region but it cannot totally remove the diamond-shaped openings between fibres induced by the stitching yarns (Fig. 2) [2]. These openings are

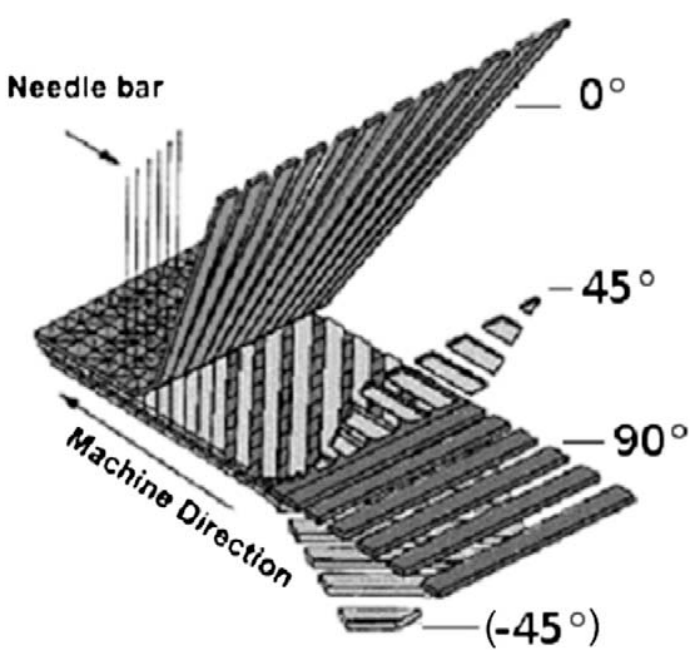

Fig. 3. NCF structure: ply assembly of a quasi-isotropic multiply [1].

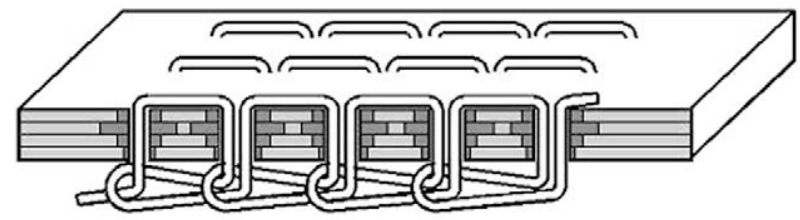

Fig. 4. Chain stitch in NCF.

of different sizes and shapes according to yarn size, the stitch type and ply orientation. The arrangement of the different plies within the stack controls the geometry of the openings and thus the resin-rich regions inside the final laminate [5]. The distance between two stitching points, referred to as the stitching step, determines the spatial period of the resin-rich region. Since the stitches in the different stacks are not aligned, the distribution of the resinrich regions throughout the laminate is not uniform. There are also interlayer gaps between the different stacks.

Quadriaxial [-45/0/45/90/90/45/0/-45 $]_{2}$ laminates were studied, with stacks composed of four plies assembled with a chain stitch (Fig. 4). Two types of stack were analyzed, a basic one [-45/0/45/90], referred to here as B, and [90/45/0/-45], referred to as $S$. The stacking sequence of the studied samples, defined as BSBS, avoids the presence of two loop sides of the stitching yarn within the same interlayer gap. The difference between the two sides of a stitched stack is shown in Fig. 5.

\subsection{Geometrical description}

The first step in modelling the mechanical or hygrothermomechanical behaviour of such a material is to build a reliable geometrical model taking into account the resin-rich region morphology. The shape of an opening in a ply is more or less similar to an el-
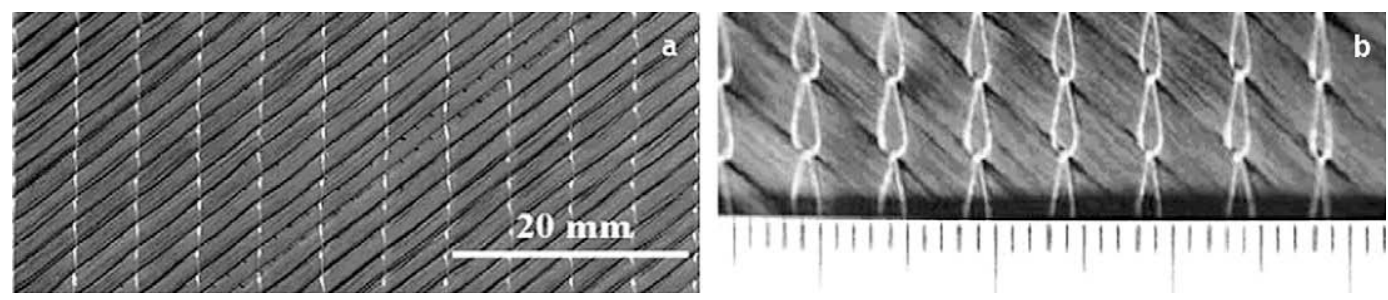

Fig. 2. NCF preform (a) and NC2 preform (b). 

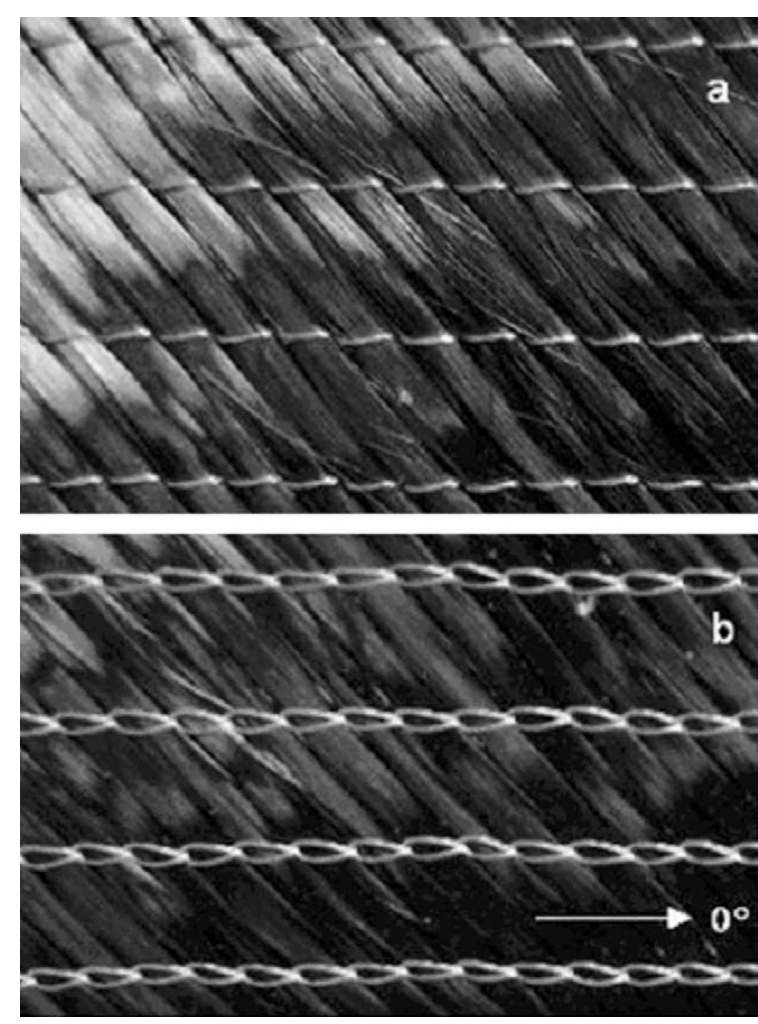

Fig. 5. Difference between the two sides of a stitched stack: the I side (a) and the loop side (b).

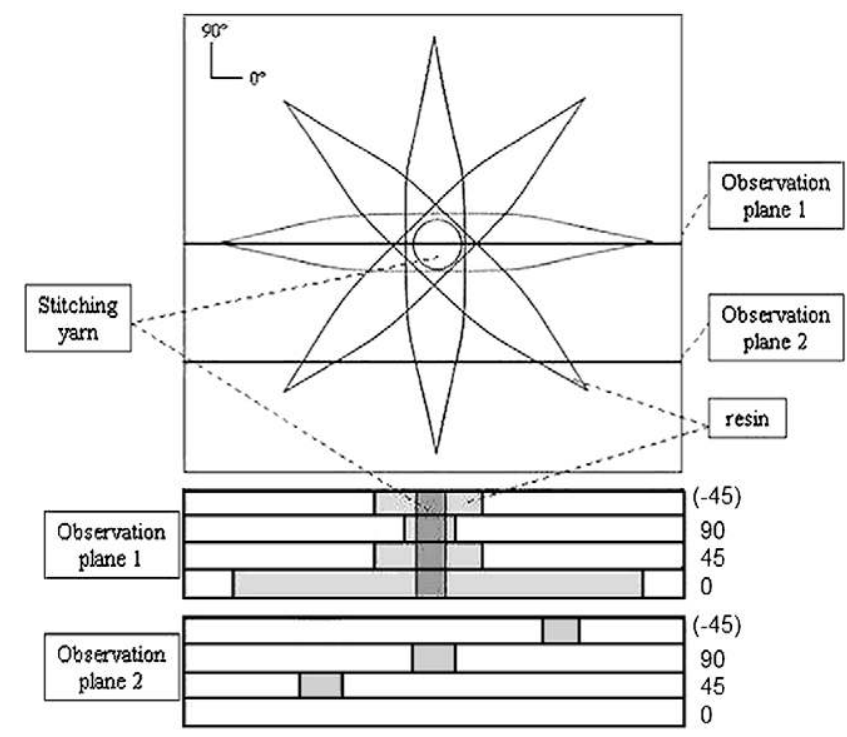

Fig. 6. Schematic view of resin-rich region in a quasi-isotropic multiply.

lipse, but the extremities of the major axis should be sharp, to represent the true opening ends. Fig. 6 shows the resin-rich regions in a quadriaxial quasi-isotropic laminate. The stitching yarn is modeled as a cylinder, even though is composed of several elementary fibres which can be detected easily by microscope. The two different cutting planes show the distribution of the resin-rich regions.

As previously pointed out, other resin-rich regions can be found between plies. The size and geometry of these regions are complex and variable; they mainly depend on stacking sequence, yarn size and stitching process. The lowest possible experimental reference

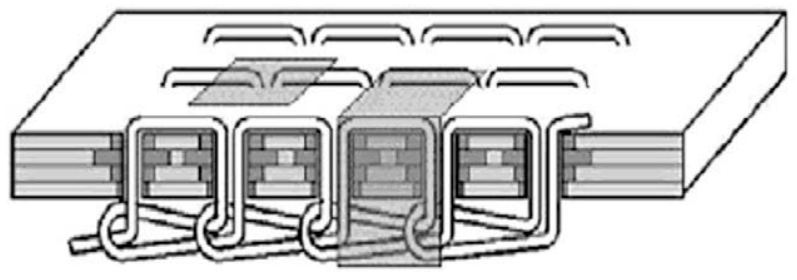

Fig. 7. Size of reference volume.

volume is needed to analyze the effects of manufacturing parameters on the morphology of materials.

\subsection{Determination of the reference volume}

The choice of the experimental reference volume should take into account the multi-scale nature of the material. The definition of the volume to be analyzed refers to the stitching yarn spacing and its minimum size is obviously that of a unit cell, i.e. the distance between two stitches along the stitching row, the distance between two consecutive stitching rows and the multi-axial stack thickness (Fig. 7). Clearly, the information included in the analysis volume does not depend on its location in a multi-axial stack because of the periodicity of the pattern (Fig. 8).

The interlayer gaps present the main difficulty in defining the reference volume because they may present very different shapes depending on loop geometry (Fig. 5). These areas will not be considered in this preliminary approach, which focuses mainly on resin channels and stitching areas.

\section{Methods of analysis}

\subsection{Optical micrography}

To characterize the geometry of the stitching area, we first used an optical micrographic technique with metallographic preparations [6]. This 2D method is destructive and requires samples to be cut and the surfaces to be carefully polished. For carbon fibre polymer matrix composite materials, we selected an abrasive cutting blade to prevent any cracks beginning and propagating near the observation surface.

The sample was then embedded in a cold-coating epoxy resin to simplify handling and allow automatic polishing. The surface to be observed was polished with decreasing grain-size abrasive papers using a Presi Mecapol P320 machine. The samples were cleaned by immersion in an ultrasound bath between each polishing step. This method has been shown to lead to a high quality surface, suitable for optical observations and characterizations (Fig. 1).

\subsection{X-ray microtomography}

Characterization by X-ray microtomography relies on a 3D reconstruction based on a series of X-ray projections and image treatments performed on a virtual sample. The X-ray beam passes through the sample and the signal transmitted is recorded by CCD sensors (Fig. 9). The X-ray-transmitted pattern is recorded for several orientations of the sample, which rotates around axis $y$. The number of patterns determines the spatial resolution of the 3D reconstruction.

Usually, conic X-ray beams are utilized in laboratory microtomographs. The voxel size to be analyzed is determined by the ratio of the distance between the source and the sample to the distance between the sample and the CCD sensor board. 


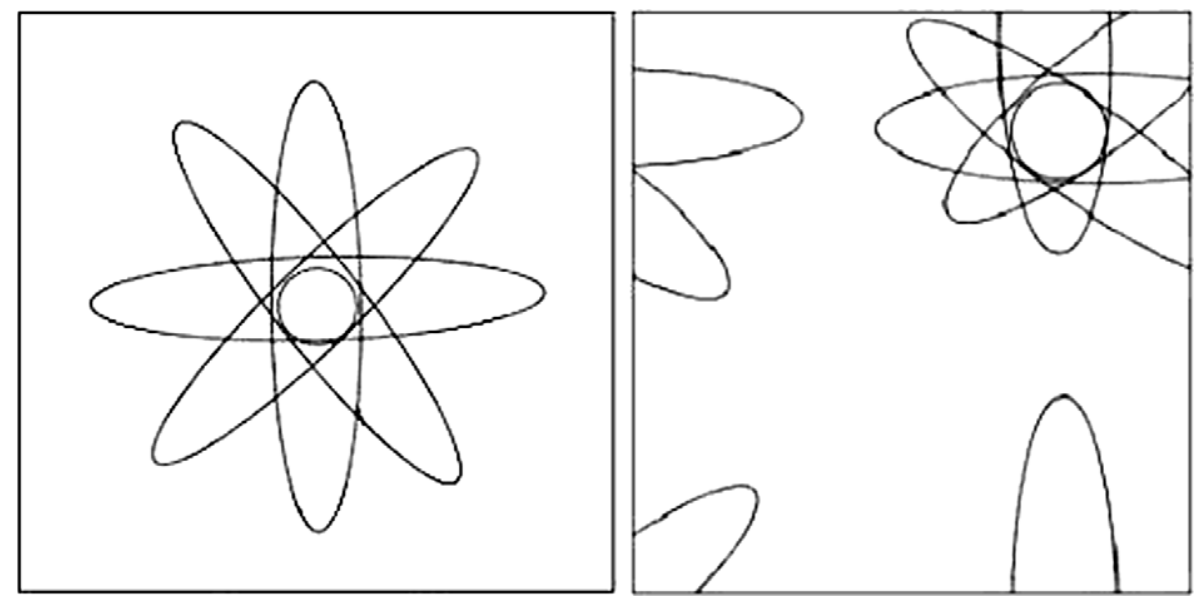

Fig. 8. Two different localisations of experimental analysis volume.

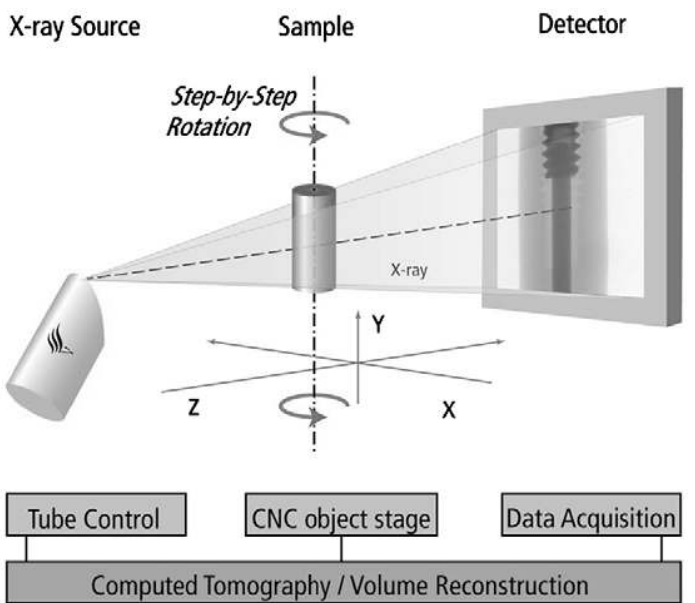

Fig. 9. X-ray microtomography equipment [7].

Here, we used a Phoenix X-ray Nanotom (USBB) for X-ray pattern acquisition. An interesting feature is that the spot on the target that characterizes the X-ray emission has an approximate diameter of $0.5 \mu \mathrm{m}$. It is one of the smallest that can be realized for laboratory use, leading to low spatial resolution. The number of CCD sensors on the board also controls the resolution of the acquired volume. The microtomograph is equipped with a square panel of 2304 by 2304 CCD sensors against 1200 by 1200 sensors in ordinary microtomographs. The combination of low spot-size and high number of sensors leads to very high resolution for a laboratory microtomograph which will be shown to be essential to detect thin microcracks in carbon fibre composites.

The reconstruction based on X-ray projections used the Phoenix $\mathrm{X}$-ray commercial software. Two thousand X-ray projection planes were used for a complete rotation of the cylindrical sample (radius: $1 \mathrm{~cm}$ ), a projection being performed every $0.18^{\circ}$ rotation. This procedure maximizes the contrast between the different areas of the sample, which is necessary to determine the resin-rich regions.

However, we wanted to enhance contrast because of the high similarities in the X-ray signatures of epoxy resin and carbon fibres.

\section{Image treatment}

Volume reconstruction requires considerable computer memory resources. Storage of the acquired data and visualization of the full reference volume is usually impossible with standard computers. There are two main procedures: either working on sub-volumes only (method used to localise the volume to be analyzed) or developing some special tools to modify the volume without any visualization, i.e. performing direct modifications of the acquired files (method used for the filtering).

After reconstruction, the 3D images of our entire sample was divided into several volumes of roughly $2000 \times 2000 \times 150$ voxels. The volumes were resized by cropping and concatenation using ImageJ software to obtain the reference volume (about $900 \times 900 \times 400$ voxels $)$.

The analysis of the virtual volume then requires advanced data treatment to reduce noise and enhance contrast for structural characterization.

\subsection{Filtering of the 3D grey level images}

An anisotropic diffusion filter was chosen for its ability to preserve edge definition. The process is based on solving the diffusion equation where the diffusion tensor is a function of the local gradient of grey level [8]. The grey levels are simply regarded as concentration levels in the diffusion equation. The classic diffusion equation of grey level $u$ writes as below:

$\frac{\partial u}{\partial t}=\operatorname{div}(\overrightarrow{\vec{D}} \cdot \operatorname{grad} u)$

If the diffusion tensor $\overrightarrow{\vec{D}}$ is isotropic, noise smoothing does not depend on the direction and edge deterioration may follow. This drawback is avoided if the diffusion tensor is anisotropic with the highest component in the orthogonal direction relative to the grey level gradient. The values of the components of the tensor must be proportional to the strength of the grey level gradient calculated with the three adjacent voxels in each direction. The fairly homogeneous zones are then smoothed and the edges are enhanced.

If the tensor can be expressed with scalars in each principal direction, we obtain Eq. (2):

$$
\frac{\partial u(r, t)}{\partial t}=\sum_{i=1}^{3} \frac{\partial}{\partial x_{i}}\left(c(r, t) \cdot \frac{\partial u(r, t)}{\partial x_{i}}\right) \quad i=1,2,3
$$

where $u(r, t)$ is the voxel grey level value at point $r$ and time $t$.

The key factor is $c(r, t)$, defined as a normalized function of the grey level gradients. Several expressions of $c(r, t)$ have been proposed $[9,10]$, and we use here the Green-Krissian form [10] because it is the simplest stable method. Stable means that noise 
cannot increase even though the parameters are arbitrarily chosen. The Green-Krissian $c(r, t)$ factor is given by Eq. (3):

$$
c(r, t)=\frac{\tanh \left(\frac{\|\operatorname{grad} u(r)\|}{k}\right)}{\frac{\| \overrightarrow{\operatorname{grad} u(r) \|}}{k}}
$$

where $k$ is a constant which can be derived from a convergence analysis. Its value has been fixed to 2 . No convergence analysis was performed because the Green-Krissian method usually ensures the stability of the process and the $k$ parameter is not of primary interest in the present work.

The problem is solved by approximation of the derivative by finite differences with the following boundary conditions [8]:

- the flux orthogonal to the edges of the domain is null and

- to interpolate the grey level gradient at the edge of the domain, virtual voxels with the same grey level as the central one are used.
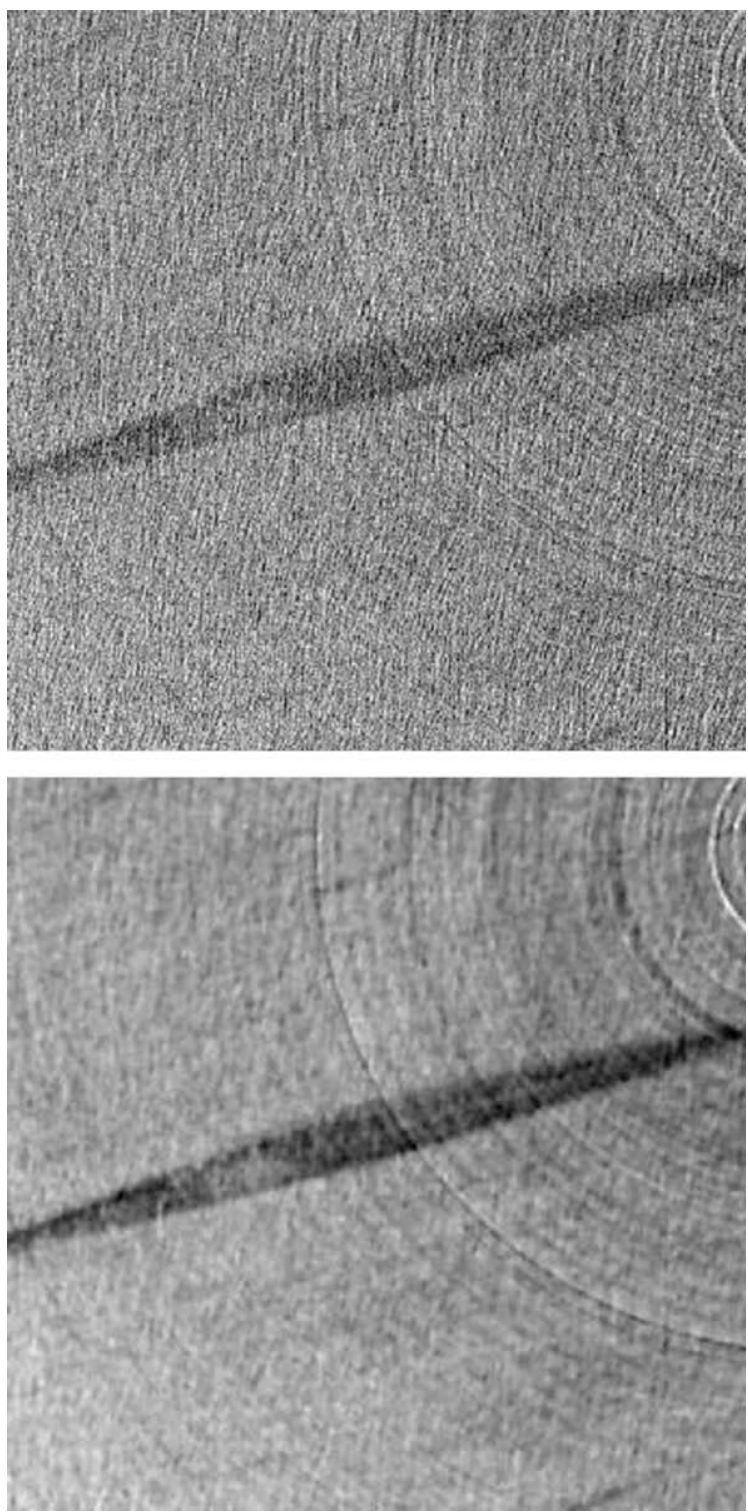

Fig. 10. Filtering effect: untreated image (up); treated image (bottom). $\mathrm{t}$ stands for the time variable in the diffusion equation. The time increment in the numerical calculation is fixed to $10 \mathrm{~s}$. A too-long diffusion time can lead to degradation in the resolution of the edges between the different parts of the material. To solve this problem, a temporary file of the volume is saved every 20 time increments. Therefore, the optimal diffusion time is still assessable even if the contrast is spoilt during image treatment. The optimal diffusion time is chosen by the operator in post-treatment. It corresponds to the volume where the edges are the smoothest after thresholding.

After filtering of the volume (Fig. 10), the resin-rich regions can be revealed using mathematical morphology tools $[11,13]$.

\subsection{Segmentation of the phases}

The filter was used to treat volumes composed of 32-bit images. Conversion to the 8-bit format was performed on only one part of the grey level spectrum. The spectrum is extended in a calibrated window during the conversion. This process induces a discretization of the spectrum and thus facilitates thresholding, the next operation. Indeed, spectrum enlargement creates bigger groups of voxels of the same grey level, separated by grey level value gaps. The threshold operation is then easier: the number of voxels selected to set a threshold remains unchanged whether or not the modification involves the bordering voxel group.

The threshold operation is a critical step in characterizing the volume of resin-rich regions, because suitable thresholding needs to be determined on the basis of several preliminary analyses. Here, the level of the threshold was close to 80 .

Classical image analysis tools derived from mathematical morphology $[11,14]$ may be used to process the 3D images. See [12] for a brief overview of tools and applications and [11,14] for more extensive and theoretical reviews. The basic operations are erosion and dilation by reference sets of given geometry called structuring elements which can be for example spheres, cubes, segments or bipoints according to desired information [11-14]. Very useful composed operations are "opening" and "closing", i.e. "erosion" followed by "dilation" and "dilation" followed by "erosion".

A difficulty arose from the similarities between the X-ray absorption of the stitching yarn and that of the carbon/epoxy composite, making it impossible to include the stitching yarn in the

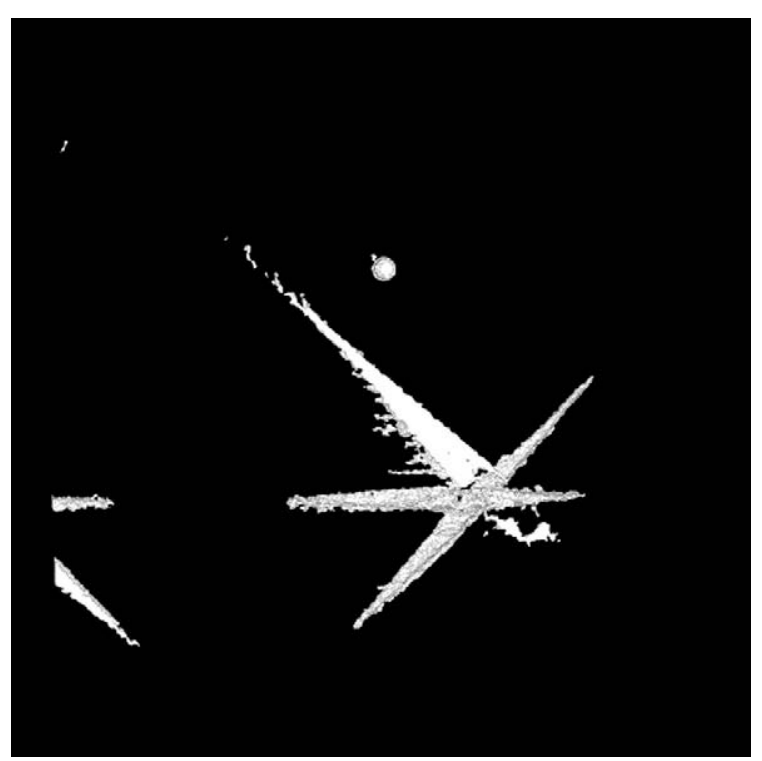

Fig. 11. Diamond-like region around the transverse stitching yarn (size in voxels: $910 \times 910 \times 180$ and resolution: $5.5 \mu \mathrm{m})$. 
resin-rich region with thresholding. The problem was solved by using the "fill holes" tool on every stack [12,13]. All closed surfaces were filled in and the holes left by yarn thresholding were filled. The process is also called "closing by reconstruction".

The previous operation raised another problem: "ring artefacts" (Fig. 10 up right and Fig. 11 centre). These well-known artefacts result from the non-homogeneity of the X-ray beam, which generates aberrant points on the CCD sensors. At the end of the rotation and reconstruction, the artefacts lead to ring formation. The "fill holes" operation fills in the closed ring artefacts. However, the ring artefacts are completely closed at the centre of the sample only. This specific effect will be analyzed in Section 5.3.

To delete the remaining noise and the unclosed ring artefacts, we used the "opening by reconstruction" tool. This operation is based on an "opening" and final image is then compared to the initial one. The parts which have disappeared remain unchanged and the volumes which have been modified, but not totally erased, are restored. If the size of the virtual particles (here an octahedron) used for the "opening" is well calibrated, this operation erases the remaining noise and the ring artefacts that have not been filled in. The diamond-like resin-rich areas after thresholding are shown in Fig. 11.

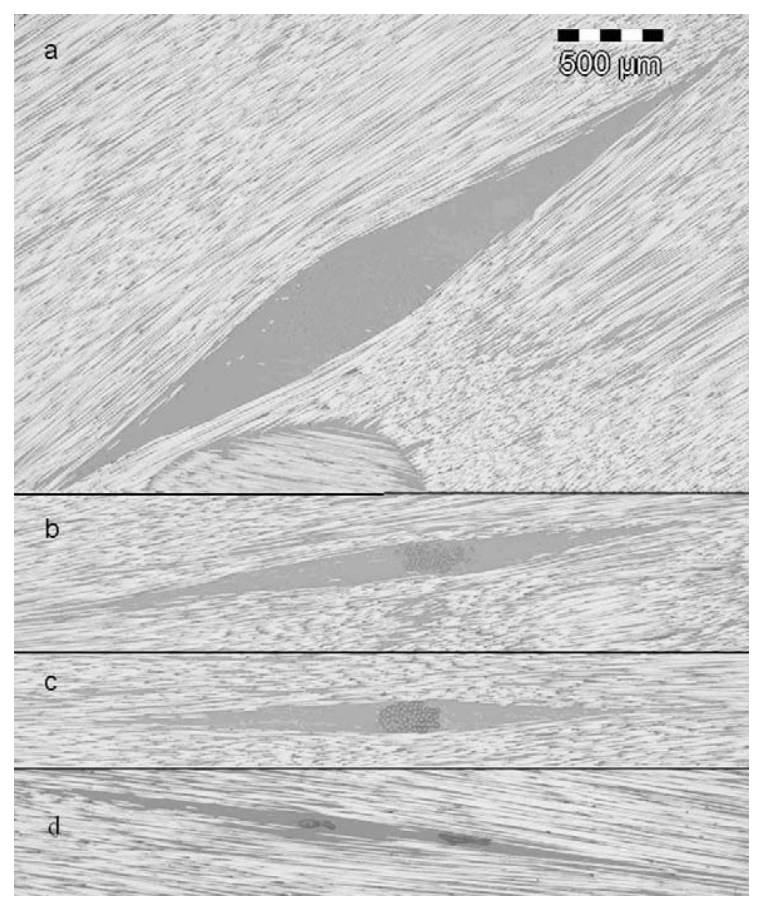

Fig. 12. Effects of diameter and tension of yarns on openings within plies: (a) yarn B; (b) loose yarn A; (c) tight yarn A; and (d) yarn C (2D destructive micrographies).

\section{Results and discussion}

\subsection{Characterization of the resin-rich region}

Our aim here was, using both 2D characterization and 3D volumes, to characterize the effects of stitching yarns of different natures, sizes and tensions. It is critical to design multi-axial multi-ply preforms taking into account such effects, to control the sizes and shapes of the resin-rich areas. Therefore, we carried out metallographic preparations with highly controlled abraded surfaces to analyze and compare similar cross-sections of samples of the same batch.

Three families of stitching yarns were analyzed: yarn A considered as a reference by the supplier (76 dTex), yarn B, which is bigger (30 Tex) and yarn C, which is thinner (23 dTex). Two types of preform were stitched with yarn A with different stitching tensions. The stitching yarn tension is defined by the length of yarn used $(\mathrm{mm})$ for manufacturing one meter of multi-axial stacks. The two tensions tested correspond to the provider reference for the tightest stitching $(3800 \mathrm{~mm} / \mathrm{m})$, referred to as tight yarn, and to the minimum tightness reachable with the manufacturing process $(4100 \mathrm{~mm} / \mathrm{m})$, referred to as loose yarn (Fig. 12).

The stacking sequence influences resin-rich region size as well. We characterized the resin-rich regions of three different multi-axial multi-ply stitched preforms: two biaxial, [90/0/0/90] and [-45/ $45 / 45 /-45]$ and a quadriaxial preform [-45/0/45/90]. For all these samples, the stitching rows are along the $0^{\circ}$ orientation. Thus the biaxial laminates differ in the orientation of the fibre relative to the stitching row. Measurements using optical micrography were carried out to quantify resin-rich region size parameters (Table 1) and their mean dispersion on five measurements (referred to as “disp").

Both the above parameters control the inclusion size; obviously the resin-rich region size increases with yarn type. Further work needs to be done to clarify any interaction between yarn size and stacking sequence on the resin-rich region size. We observed in $2 \mathrm{D}$ micrograpies that the stitching row orientation has a significant effect. The relationship between the parameters became more complex in 3D and departures from the ideal geometrical model were observed. This point will be discussed in the next section.

The dissymmetry of the resin channels should be emphasized. Fig. 12 shows that tight yarns tend to lead to a higher dissymmetry. For instance, the ratio of the distances between the stitching yarn and each end of a transverse channel is around 0.9 for tight stitching yarns and can reach 0.6 for loose ones. This tendency needs to be validated in further studies since it may in part be due to manufacturing processes. The dissymmetry has a critical effect on the $3 \mathrm{D}$ geometry of the resin-rich regions. A typical example of a quadriaxial preform with significant dissymmetry in every ply is shown in Fig. 13.

Table 1

Opening parameters obtained by 2D observation.

\begin{tabular}{|c|c|c|c|c|c|c|c|c|c|c|}
\hline \multirow[t]{2}{*}{ Yarn type } & \multicolumn{2}{|c|}{ Major axis } & \multicolumn{2}{|c|}{ Minor axis $₫$} & \multicolumn{2}{|c|}{$\underline{\text { Yam size } \Theta \Theta}$} & \multicolumn{4}{|c|}{ Dissymmetry: distances ends/yarn $\longleftrightarrow$} \\
\hline & $\mathrm{mm}$ & disp & $\mathrm{mm}$ & disp & $\mathrm{mm}$ & disp & $\mathrm{mm}$ & disp & $\mathrm{mm}$ & disp \\
\hline \multicolumn{11}{|l|}{$[135 / 0 / 45 / 90]$} \\
\hline Yarn A tight & 2.2 & 0.2 & 0.18 & 0.02 & 0.2 & 0.1 & 1.3 & 0.3 & 1 & 0.2 \\
\hline Yarn A loose & 2.6 & 0.2 & 0.16 & 0.02 & 0.29 & 0.05 & 1.5 & 0.2 & 1.1 & 0.2 \\
\hline Yarn B & 3.6 & 0.2 & 0.45 & 0.03 & 0.65 & 0.05 & 2.1 & 0.2 & 1.5 & 0.1 \\
\hline Yarn C & 2.5 & $0_{\mathrm{r}} 4$ & 0.1 & 0.07 & 0.18 & 0.01 & 1.4 & 0.1 & 1.1 & 0.1 \\
\hline \multicolumn{11}{|c|}{$[135 / 45 / 45 / 135]$} \\
\hline Yarn B & Channels & & 0.47 & 0.1 & 1 & 0.2 & Channels & & & \\
\hline \multicolumn{11}{|l|}{$[90 / 0 / 0 / 90]$} \\
\hline Yarn B & Channels & & 0.91 & 0.18 & 1.1 & 0.2 & Channels & & & \\
\hline
\end{tabular}




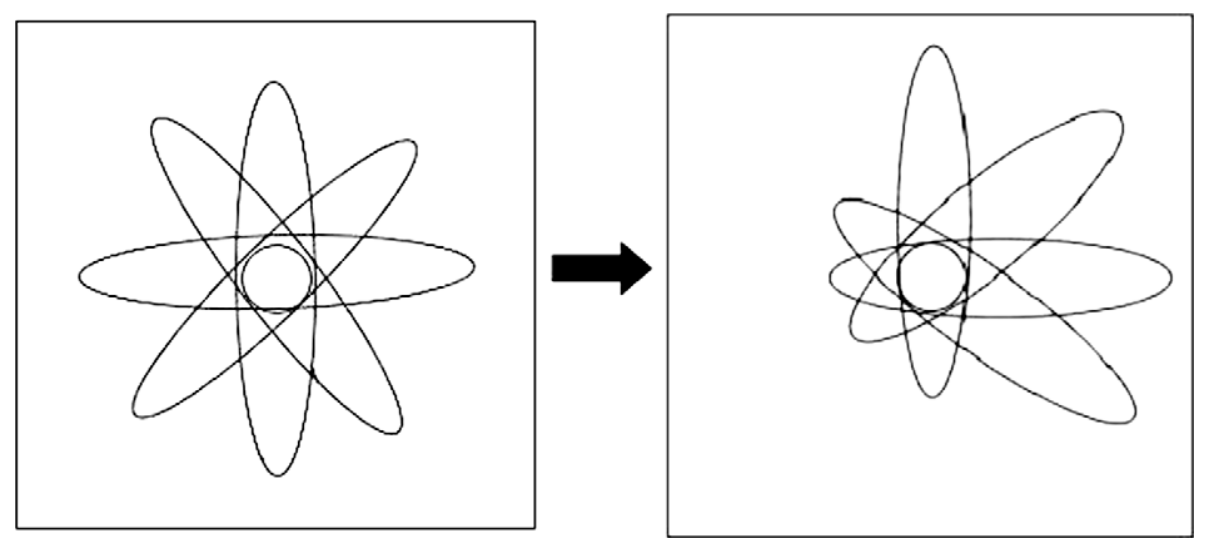

Fig. 13. Distorsion of diamond-like regions.

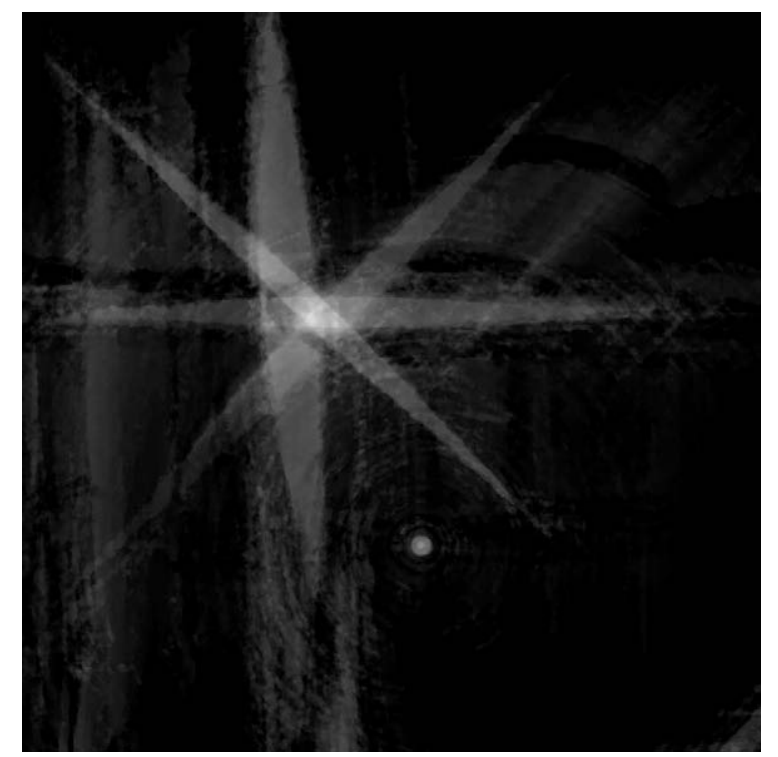

Fig. 14. Average projection of a treated analysis volume $\left(5 \times 5 \times 1 \mathrm{~mm}^{3}\right)$.

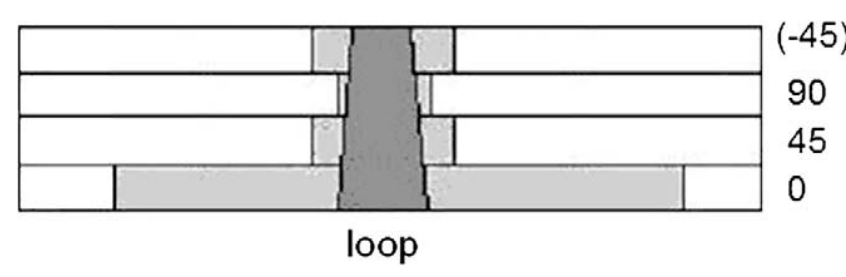

Fig. 15. Conical shape of transverse channel due to loop.

Table 2

Calculation of the inclusion volume ratio in percent, function of the type of image treatment.

\begin{tabular}{lllll}
\hline $\begin{array}{l}i \cdot j \text { Sample } i \\
\text { easurement } j\end{array}$ & $\begin{array}{l}\text { Entire } \\
\text { treatment }\end{array}$ & $\begin{array}{l}\text { Mean } \\
\text { variation }\end{array}$ & $\begin{array}{l}\text { Without } \\
\text { opening }\end{array}$ & $\begin{array}{l}\text { Mean } \\
\text { variation }\end{array}$ \\
\hline 1.1 & 3.60 & 0.56 & 3.80 & 0.76 \\
1.2 & 4.10 & 1.06 & 4.60 & 1.56 \\
2.1 & 2.70 & 0.34 & 3.40 & 0.36 \\
2.2 & 3.00 & 0.04 & 3.70 & 0.66 \\
3.1 & 2.60 & 0.44 & 3.00 & 0.04 \\
3.2 & 2.25 & 0.79 & 2.95 & 0.09 \\
Average & 3.04 & 0.54 & 3.58 & 0.58 \\
\hline
\end{tabular}

\subsection{Geometrical description correction}

Using 3D observations, two main changes were introduced into the geometrical model detailed in Section 2.2, involving modification of yarn shape and its effects on the resin-rich region morphology. The effects of the yarn can be characterized by analyzing either several stacks or the projection of all the X-ray patterns in the transverse direction. This projection can be performed by averaging the grey level of the column of voxels which are aligned in the projection direction, so that, each pixel of the projection represents the average of the grey levels of the volume in the ply transverse direction (Fig. 14).

The first conclusion is that the minor axes of the openings are not constant for a given stitching yarn. They tend to gradually increase, reaching a maximum on the loop side. For a quadriaxial preform stitched with tight $A$ yarn, the minor axis value varies from $0.15 \mathrm{~mm}$ on side I to $0.4 \mathrm{~mm}$ on the loop side. This phenomenon is regarded as an enlargement of the stitching yarn (Fig. 15).

The second conclusion is that the stitching yarn is not normal to the plies. 3D analysis makes it possible to assess the angle between the yarn and the perpendicular to the ply. The mean angle for the tight $A$ yarn in quadriaxial is $25^{\circ}$ with a scatter of $\pm 5.5^{\circ}$.

These two conclusions indicate some scatter of properties depending on the process. They also prove that a 3D characterization of the geometry is required for a reliable morphological model, a pre-requisite to any further material behaviour modelling.

\subsection{Volume fraction occupied by the resin-rich region}

Characterizing of the resin-rich regions by thresholding enables us to measure the volume of resin-rich regions within the analysis volume, and thus determine the volume fraction occupied by resin-rich regions in the entire material. Measurements were made on three different specimens composed of quadriaxial preforms stitched with A yarn. Two stitches on each specimen were analyzed. Moreover, to estimate the effect due to the "rebuilt opening" and different image treatments, which control the reliability of measurements, the sizes of the resin-rich regions were also estimated without any mathematical morphology operation (Table 2).

The average resin volume fraction in these regions is $3 \%$ for the specimens. The mean variation is about $0.5 \%$ which indicates that the measurement method is reasonably accurate, taking into account the difficulty of data treatment. Table 2 shows that the "rebuilt opening" which erases residual noise has no significant effect on the mean variation. It only tends to slightly increase the resin volume fraction. It seems to us that this operation does not 

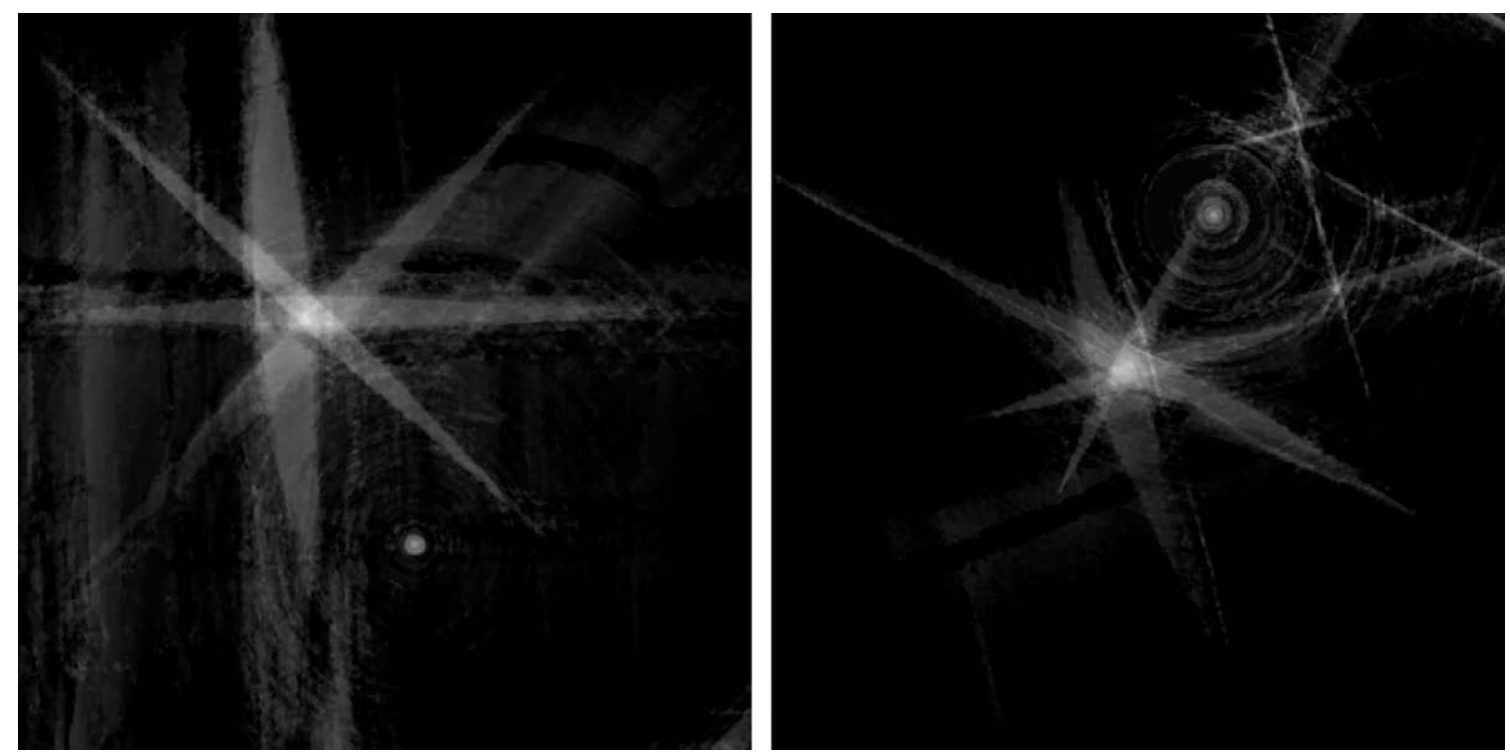

Fig. 16. Examples of two projections of resin-rich regions from two different samples.

significantly perturb the analysis, since the difference remains of the order of the experimental scatter.

Tests were also carried out to check the influence of the other operations of mathematical morphology, which were shown to have no significant effect on either volume fraction or mean variation. Basically, the main source of variation is thresholding. The spectrum enlargement during conversion from 32 bits to 8 bits minimizes errors due to thresholding, but it is still the key step in image treatment. The measurement of the same resin volume with three different thresholds where a grey level group was included or excluded led to an average value of $3 \%$ with a mean difference of $\pm 3 \%$. This clearly confirms that a grey level threshold calibration taking account of the entire range of analysis volumes involved in the study is absolutely essential.

It is interesting to note that the measurement scatter between specimens from two different batches is significantly higher than that from the same batch. This effect proves that control of infusion conditions should be improved (Fig. 16)

The share of the interlayer gaps in the resin volume fraction is about $1 \%$ of the entire material volume, namely only a third of the resin-rich region. As previously mentioned, these interlayer gaps can show very different geometries that cannot be predicted at the moment.

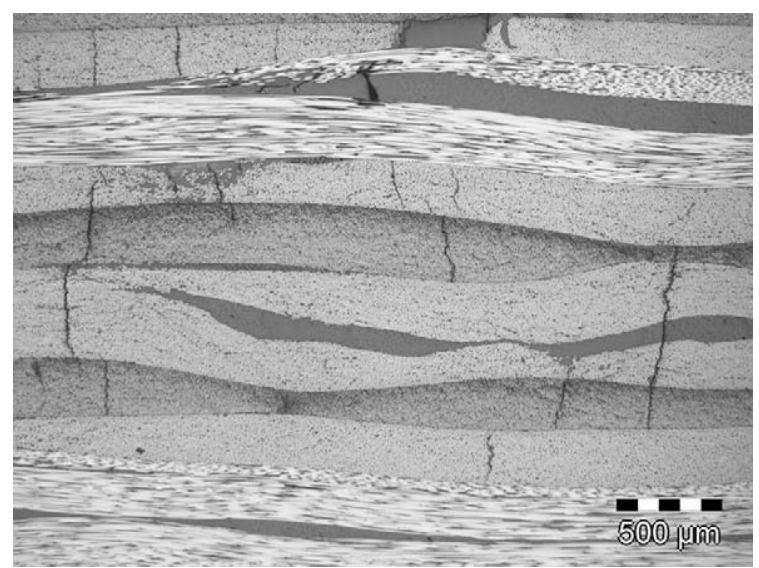

Fig. 17. Hygrothermal microcracks from cyclical loading.
To conclude on resin volume fraction assessment, we developed an interesting approach to quantify the volume fraction of the resin-rich region and finally obtained $3 \% \pm 0.5 \%$ for quadriaxial preforms stitched with A yarn. These specimens have a fibre volume fraction of $67 \%$ measured by resin digestion. This means that the resin-rich regions represent $9 \%$ of the total resin volume of the composite. Such resin concentrations may have a significant effect on the physical and mechanical behaviour of the material, particularly with respect to hygrothermal loading fatigue.

\subsection{Hygrothermal microcracks}

3D imaging opens the way to the characterization of multiscale morphologies inside composite materials, as discussed in the preceding section. However, it can also be used to detect the start and spread of damage. In this section, we show that microcracks induced by hygrothermal fatigue can be revealed [15-19] (Fig. 17). The hygrothermal loading is composed of 2000 cycles of $1 \mathrm{~h}$. The extremum temperatures, $80^{\circ} \mathrm{C}$ and $-55^{\circ} \mathrm{C}$, are maintained during $15 \mathrm{~min}$ in each cycle.

The crack opening varies between 5 and $10 \mu \mathrm{m}$. The minimum spatial resolution for a volume is two voxels. We took the decision to acquire a volume in local microtomography with a voxel size of
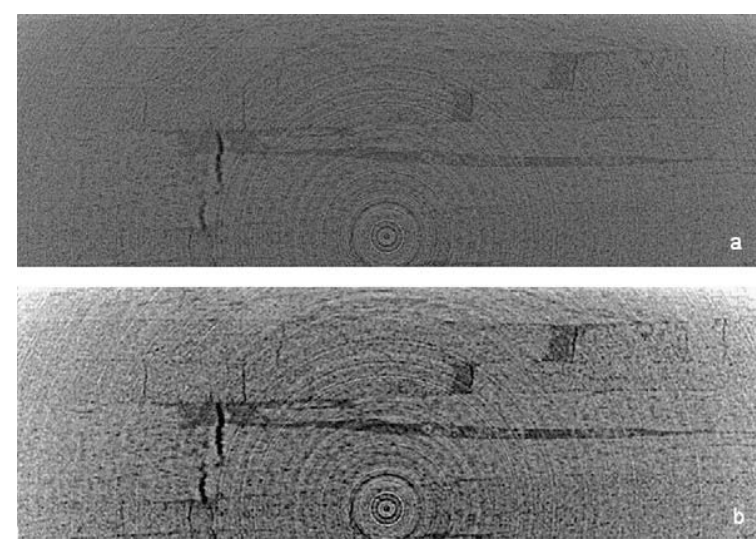

Fig. 18. Section of a cracked sample obtained by local microtomography before (a) and after (b) filtering. 
$2.5 \mu \mathrm{m}$. The unique feature of local microtomography is that only one part of the specimen is acquired and studied. This approach prevents any calibration of the non-attenuated beam of the X-ray projections. Local microtomography leads to lower spatial resolution but it induces higher noise on the volumes. The volumes are treated with the filter previously discussed (Section 4.2) (Fig. 18).

The cracks in a resin-rich region can be detected by thresholding but it is very difficult to visualize the entire crack network (Fig. 19). One solution is to analyze the projections transverse direction from the plies. Again the average of the voxel grey level values reveals local information if the projection is restricted to a single multi-axial stack (Fig. 20). Here, projecting the lowest grey level along the transverse direction avoided limitation to a single

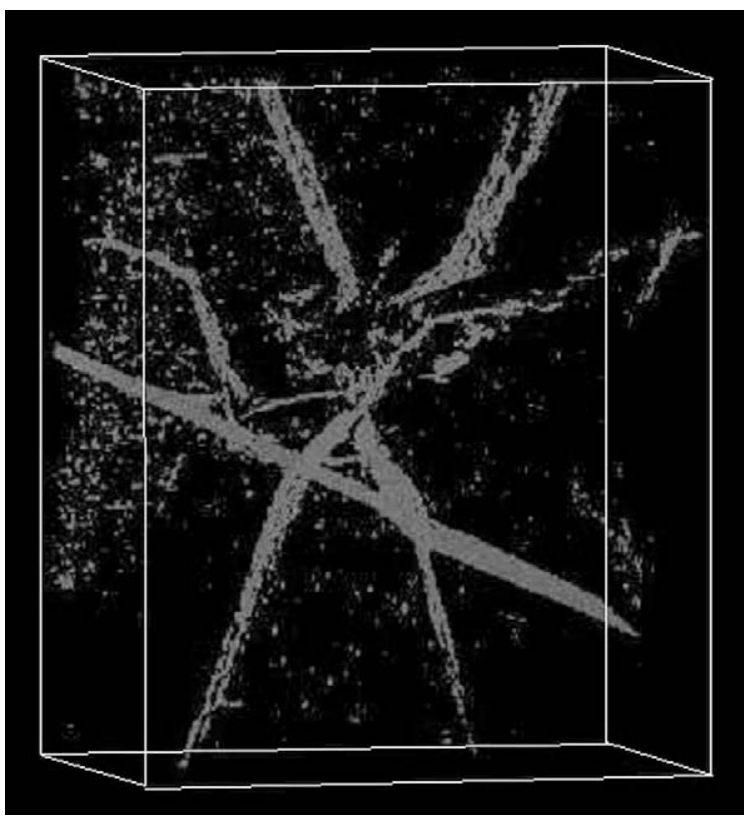

Fig. 19. 3D rebuilt microcrack after threshold in a resin-rich region.

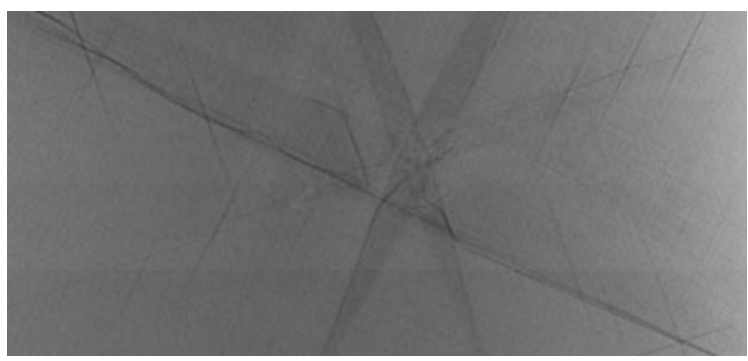

Fig. 20. Detection of microcracks: average projection of a single multi-axial stack.

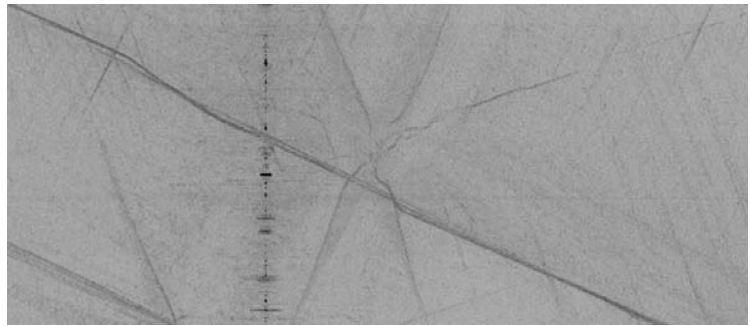

Fig. 21. Detection of microcracks: projection of the minimum grey level of a single multi-axial stack.

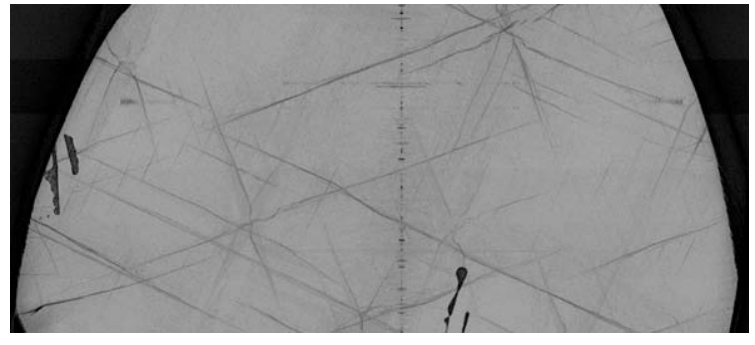

Fig. 22. Detection of microcracks: projection of the minimum grey level of four multi-axial stacks.

multi-axial stack (Fig. 21). The projection of the 3D crack network inside standard specimens was thus visualized (Fig. 22). The 3D method developed in this study allows the characterization of the three-dimensional crack network, and could be useful to monitor the cracking of specimens during an ageing process. Quantification of cracks and comparison between the 3D and traditional 2D destructive methods will be presented in a further publication focused on durability of NC2 under cyclical hygrothermal loading.

\section{Conclusion}

The morphology of the new NCFs is complex, and the optimisation of those materials requires advanced approaches based on 3D observations in addition to the common 2D destructive observations, which only provide a narrow insight into the meso- and micro-structures of such materials. Our results indicate that X-ray microtomography appears better suited to analysis of these materials because their micro-structures are fundamentally three dimensional.

This method is also promising for the study of cracking mechanisms such as hygrothermal microcracking. The connectivity of the crack network and the influence of resin-rich regions on crack propagation can be characterized by using X-ray microtomography, opening the way to the study of the growth of microcracks over time in these families of materials and the possible damaging interactions with resin-rich regions. All this is possible because $\mathrm{X}$-ray microtomography is a non-destructive method. In a future article, we will present the results of our second experimental comparison of 2D destructive and 3D microtomography monitoring of damage to samples from typical hygrothermal cyclical fatigue.

\section{Acknowledgments}

The authors wish to thank HEXCEL Corporation S.A.S. for reinforcement supply and for manufacturing the materials (Liquid Resin Infusion). A special "thank you" to Jérôme Malvestio who processed the acquisition of the microtomographic volumes and shared his expertise with us.

\section{References}

[1] Liotier PJ, Vautrin A, Beraud JM, Henrat P. Introduction to the characterization of hygrothermal microcracking of crossply composites reinforced by stitched non-woven UD laminae. Appl Mech Mater 2008;13-14:77-83.

[2] Lomov SV, Belov EB, Bischoff T, Ghosh SB, Truong Chi T, Verpoest I. Carbon composites based on multiaxial multiply stitched preforms. Part 1: geometry of the perform. Composites Part A 2002;33:1171-83.

[3] Drapier S, Pagot A, Vautrin A, Henrat P. Influence of the stitching density on transverse permeability of non-crimped new concept (NC2) multiaxial reinforcements: measurements and predictions. Compos Sci Technol 2002;62:1971-91.

[4] Reynolds TG, McManus HL. Accelerated tests of environmental degradation in composites materials. In: Grand $\mathrm{P}$, Rousseau $\mathrm{CQ}$ editors. Composites 
structures: theory and practice. West Conshohocken (PA): ASTM STP 1383, American society for Testing and Materials. p. 513-25.

[5] Chun HJ, Kim HW, Byun JH. Effects of through-the-thickness stitches on the elastic behavior of multi-axial warp knit fabric composites. Compos Struct 2006;74:484-94.

[6] Hayes BS, Gammon LM. Composites. ASM Handbook 2001;21:964-72.

[7] Phoenix X-ray system and service, Operation manual. Data X-reconstruction; 2006.

[8] Weickert J, Ter Haar Romeny BM, Viergever MA. Efficient and reliable schemes for nonlinear diffusion filtering. IEEE Trans Image Process 1998;7(3):398-410.

[9] Perona P, Malik J. Scale-space and edge detection using anisotropic diffusion. IEEE Trans Pattern Anal Mach Intell 1990;12(7):629-39.

[10] Krissian K, Malandain G, Ayache N. Directional anisotropic diffusion applied to segmentation of vessels in 3D images. Technical Report 3064, INRIA; 2001.

[11] Serra J. Image analysis and mathematical morphology, vol.1. Olando (FL, USA): London academic Press; 1982.
[12] Coster M, Chermant JL. Précis d'analyse d'images. Presses du CNRS; 1989.

[13] Soille P. Morphological image analysis principles and applications. 2nd ed. Berlin: Spinger Verlag; 1999.

[14] Matheron G. Eléments pour une théorie des milieux poreux. Paris: Masson; 1967.

[15] Liotier PJ. Microcracking of composites reinforced by multiaxial multiply stitched preforms under cyclical hygrothermal loading. PhD thesis. Ecole des Mines de Saint-Etienne; 2008.

[16] Mattsson D, Joffe R, Varna J. Damage in NCF composites under tension: effect of layer stacking sequence. Eng Fract Mech 2008;75(9):2666-82.

[17] Park CH, MacManus HL. Thermally induced damage in composite laminates: predictive methodology and experimental investigation. Compos Sci Technol 1996;56:1209-19.

[18] Nickerson S, Mayes JS, Welsh JS. Multi-continuum analysis of thermally induced matrix cracking. Eng Fract Mech 2005;72:1993-2008.

[19] Aymerich F, Priolo P, Sun CT. Static and fatigue behaviour of stitched graphite/ epoxy composite laminates. Compos Sci Technol 2003;63:907-17. 